\title{
Forward Adaptive Dual-Mode Quantizer Based on the First-Degree Spline Approximation and Embedded G.711 Codec
}

\author{
Zoran PERIC ${ }^{1}$, Jelena NIKOLIC ${ }^{1}$, Bojan DENIC ${ }^{1}$, Vladimir DESPOTOVIC ${ }^{2}$ \\ ${ }^{1}$ Dept. of Telecommunications, Faculty of Electronic Engineering, University of Nis, A. Medvedeva 14, 18000 Nis, Serbia \\ ${ }^{2}$ Technical Faculty in Bor, University of Belgrade, Vojske Jugoslavije 12, 19210 Bor, Serbia \\ \{zoran.peric, jelena.nikolic, bojan.denic\}@elfak.ni.ac.rs, vdespotovic@tfbor.bg.ac.rs
}

Submitted July 16, 2019 / Accepted October 1, 2019

\begin{abstract}
In this paper, we propose a novel model of dualmode quantizer that combines the restricted and unrestricted forward adaptive piecewise linear scalar quantizers based on the first degree-spline functions, one of them being forward adaptive G.711 quantizer used as the unrestricted one. The analysis presented in the paper can be considered as our further research in the field of dualmode quantization. In particular, in our novel model we utilize G.711 codec due to the compatibility reasons and we develop one completely novel model of restricted quantizer based on the first-degree spline approximation, which is optimized for the assumed Laplacian source so that to provide a minimal mean-squared error distortion. Moreover, unlike previous dual-model quantizer models that processed signals in frame-by-frame manner, our novel model utilizes frame/subframe processing of the signal in order to decrease the total bit rate. The theoretical analysis in a wide dynamic range of input signal variances reveals that the proposed model of quantizer is superior versus the unrestricted G.711 quantizer as well as other similar baselines having the same number of quantization levels. In addition, the results of the experimental analysis performed on the real speech signal show a good agreement with the theoretical ones.
\end{abstract}

\section{Keywords}

Scalar quantization, restricted quantization, dualmode quantization, Laplacian source, SQNR

\section{Introduction}

Quantization refers to the process of representing a large (possibly infinite) set of values with a finite (preferably small) set of discrete values [1]. In scalar quantization, each discrete sample is individually processed [1], [2]. An important variant of scalar quantization is a quantization based on switching, where there is a set of scalar quantizers at disposal and switching among them is ena- bled according to the specific rule [1]. Scalar quantizers can be categorized into the uniform and non-uniform ones. Unlike uniform quantizers that are simple for design [1-4], the design of non-uniform quantizers is rather more complex and assumes application of iterative Lloyd-Max's algorithm or companding technique [1]. The companding technique is commonly used in the design of high-rate nonuniform scalar quantizers (a cascade connection of compressor, uniform quantizer and expandor), since the LloydMax's algorithm is time-consuming.

Different solutions based on switched quantization using non-uniform quantizers have been proposed for effective processing of non-stationary signals (such as speech signal), e. g. [5]. Since in that case the variance (power) is a changeable quantity, the challenge is to maintain robustness, i.e. to provide an approximately constant Signal to Quantization Noise Ratio (SQNR) over the entire variance range of interest. In order to efficiently process time-varying signals, it has been recommended to use short intervals denoted as frames, since in that case the signal properties remain almost unchanged [1], [2]. The quantizer based on switching proposed in [5] consists of $k(k>1)$ fixed-rate $\mu$ law companding quantizers, for frame-by-frame processing of signals following the Laplacian distribution. The robustness has been achieved by dividing the dynamic range of the signal variance into a number of subranges, with one $\mu$ law companding quantizer designed for each subrange. Selection among the quantizers has been done according to the estimated frame variance; hence, the adaptation to the variance has been performed. The simplification of the mentioned switched quantization scheme with $k$ specially designed scalar quantizers can be obtained by using the forward adaptive scalar quantizer with $k$-levels log-uniform quantizer for frame variance quantization (the parameter used to scale the adaptive codebook), where exactly the same performance can be achieved [1].

The high-rate scalar quantization solutions, representing the combination of forward adaptive technique and switched technique have been proposed in [6-8]. In these works, for processing of each frame, one of two available 
forward adaptive companding scalar quantizers, restricted or unrestricted, has been used, hence the name dual-mode scalar quantization. In particular, the employed quantizers have similar characteristics, the same number of quantization levels $N$, but unequal support regions. Recall that the restricted quantizer has been obtained as the result of restricting the bounds of the support region, which inserts granular distortion and eliminates overload distortion. With the properly chosen support region threshold, which is of extreme importance [9], [10], an $\mathrm{N}$-levels restricted quantizer provides substantially increased quantization level density compared to the $N$-levels unrestricted one, and enables better quantization (i.e. smaller quantization error). The main characteristic of dual-mode quantization is its ability to provide increased performance (SQNR) over the single fixed-rate (unrestricted) quantizer, by providing more frequent selection of the restricted quantizer. In [6-8], the selction among the quantizers has been performed framewise, i.e. the restricted one has been chosen for frames whose amplitude content is within its support region, while for other frames the unrestricted one has been employed.

A model of quantizer which combines the forward adaptive companding quantizers with nonlinear $\mu$-law compression functions for quantization of the Laplacian sources has been proposed in [6]. A quantizer model in [7] has also been developed for the Laplacian source, but it utilizes the forward adaptive companding quantizers based on the optimal compression function. Unlike [6] and [7], the authors in [8] have proposed a quantization solution for coding the Gaussian source, consisted of forward adaptive piecewise linear companding quantizers (PLCSQs) obtained as the result of linearization of optimal compression function. The design goal in the above mentioned quantization schemes were reducing the bit rate subject to the constraint that high quality coding is enabled. Furthermore, a non-adapive (designed for the particular variance and the Laplacian source) dual-mode quantization model that uses a $\mu$-law scalar companding quantizer as a restricted quantizer and G.711 quantizer [11] as the unrestricted quantizer has been proposed in [12].

This paper proposes a novel model of dual-mode quantizer with embedded G.711 quantizer for the Laplacian source that is further implemented in forward adaptive scheme (i.e. forward adaptive dual-mode scalar quantizer (DMSQ)). Particularly, the aim of this paper is to enhance the performance of the G.711 codec [11] in a wide range of input signal variances, achieveable by using adaptation [13]. The performance improvement of that extensively used codec has been the subject of various papers, e.g. [14], [15]. In [14], an enhancement has been achieved in the context of log-polar quantization, while in [15] an enhancement approach based on a convolutional neural network has been presented. Eventually, we should highlight here that high rate codecs such as G.711 codec can be considered as a good candidate for compression purposes in neural networks [16-18].
In regard to the previous DMSQ solutions [6-8, 12], our novel model uses the spline functions [19] and frame/subframe method to process the input signal. Firstdegree spline function is used to approximate the nonlinear $\mu$-law compression function, which greatly simplifies the practical realization of the compression function. Hence, the model we propose consists of two (restricted and unrestricted) PLCSQs, one of them being G.711 quantizer used as the unrestricted one. A new frame/subframe method implies division of the input samples into the frames of $M_{0}$ samples and forming the subframes of length $M$ within each frame for selection purposes. Using the novel method, two adaptations are encountered: at the frame level an adaptation to variance is performed and at the subframe level an adaptation to maximum amplitude is perfromed (choosing one of two available forward adaptive quantizers). In this paper, the frame length is much smaller then the ones used in [6-8] where frame-by-frame signal processing has been performed, which is not efficient enough in terms of bit rate, so that the solution we propose improves the models from [6-8]. Moreover, by using subframes instead of entire frames, more frequent selection of the restricted quantizer is assured, and, accordingly, the increase of the overall performance is expected. This is obtained at the expense of slightly increased bit rate, since the information about the frame variance and employed quantizer $\left(M_{0} / M\right.$ one-bit codewords) has to be sent to the decoder once per frame.

In brief, the solution we propose provides the following advantages over the single G.711 quantizer [11]:

- it can offer approximately constant SQNR in the entire range of the input signal variances;

- for only $0.1875 \mathrm{bit} / \mathrm{sample}$ increased bit rate, it can achieve the gain in maximum SQNR of approximately $6 \mathrm{~dB}$ and the gain in the average SQNR of approximately $11.3 \mathrm{~dB}$;

- it can provide gain in average SQNR of approximately $5 \mathrm{~dB}$, with compression of approximately $0.8125 \mathrm{bit} / \mathrm{sample}$.

Furthermore, the increased performance in terms of average SQNR over similar forward adaptive solutions having the same number of levels is also indicated. In addition to the theoretical results, which have been provided in the majority of DMSQ models developed so far $[6,8$, 12], an experimental analysis using speech signal extracted from the TIMIT database [20] is provided in this paper as well, which is an additional progressive step with respect to former solutions.

The remaining of the paper is organized as follows: in Sec. 2, a detailed design procedure of the employed PLCSQs, forming the core of the proposed DMSQ, is described. Section 3 deals with the implementation in the forward adaptive DMSQ. Section 4 summarizes and discusses numerical and experimental results. Section 5 concludes the paper. 


\section{Design of PLSQ Based on First- Degree Spline Functions}

PLCSQ is based on the piecewise linear compression function, used as an alternative step to nonlinear compression function. We propose fixed-rate $N$-levels PLSCQ based on the first-degree spline functions, when the signal to be quantized is described by the memoryless Laplacian source having PDF [1], [2]:

$$
p(x, \sigma)=\frac{1}{\sqrt{2} \sigma} \exp \left(-\frac{\sqrt{2}|x|}{\sigma}\right)
$$

where $\sigma^{2}$ is the variance of the signal. The design of PLCSQ is performed for one particular (reference) variance $\sigma_{\text {ref }}^{2}$, using $p\left(x, \sigma_{\text {ref }}\right)$.

\subsection{The First-Degree Spline Functions}

Spline refers to a piecewise polynomial approximation of the mathematical function [19]. Generally, using spline, the approximation of the function can be achieved by division of interval of interest into a finite integer number of $L$ segments $\left[x_{i}, x_{i+1}\right], i=0, \ldots, L-1$, where within each segment the function is approximated by the corresponding polynomial. $x_{i}=x_{i}\left(\sigma_{\text {ref }}\right), i=0, \ldots, L$, are called 'knots' representing the points where the function changes its character. In this paper, we use the simplest version of spline, i.e. the first-degree spline (it is composed of straight line segments that are connected in a way to form continuality), to approximate the logarithmic $\mu$-law compression function, $c\left(x, \sigma_{\mathrm{ref}}\right)$, defined by [1]:

$$
c\left(x, \sigma_{\text {ref }}\right)=\left\{\begin{array}{c}
\ln \left(1+\mu \frac{x}{x_{\max }}\right) \\
x_{\max } \frac{\ln (1+\mu)}{\ln \left(1-\mu \frac{x}{x_{\max }}\right)},-x_{\max } \leq x \leq 0 \\
x_{\max } \frac{\ln (1+\mu)}{\ln \left(1+x_{\max }\right.}
\end{array}\right.
$$

where $\mu$ is the compression factor and $x_{\max }=x_{\max }\left(\sigma_{\mathrm{ref}}\right)$ is the upper support region threshold of the quantizer designed for $p\left(x, \sigma_{\text {ref }}\right)$. Due to the symmetry, only positive part $\left[0, x_{\max }\right]$ is considered.

The first-degree spline function, $c_{\mathrm{PLC}}\left(x, \sigma_{\mathrm{ref}}\right)$, employed to approximate $c\left(x, \sigma_{\text {ref }}\right)$ on $\left[0, x_{\max }\right]$ is defined as [19]:

$$
c_{\mathrm{PLC}}\left(x, \sigma_{\mathrm{ref}}\right)=\left(\begin{array}{l}
c\left(x_{1}\right)+m_{1}\left(x-x_{1}\right), x \in\left[x_{0}=0, x_{1}\right] \\
c\left(x_{2}\right)+m_{1}\left(x-x_{2}\right), x \in\left[x_{1}, x_{2}\right] \\
\vdots \\
c\left(x_{L-1}\right)+m_{L}\left(x-x_{L-1}\right), x \in\left[x_{L-1}, x_{\max }\right]
\end{array}\right.
$$

where $m_{i}, i=1, \ldots, L$, are the coefficients of direction determined as [1]:

$$
m_{i}=\frac{c\left(x_{i}, \sigma_{\mathrm{ref}}\right)-c\left(x_{i-1}, \sigma_{\mathrm{ref}}\right)}{x_{i}-x_{i-1}}, i=1, \ldots, L
$$

We derive $x_{i}$ from the following condition [1]:

$$
c\left(x_{i}, \sigma_{\mathrm{ref}}\right)=i \frac{x_{\max }}{L}, i=0,1, \ldots, L,
$$

which results in:

$$
x_{i}=\frac{x_{\max }}{\mu}\left((1+\mu)^{\frac{i}{L}}-1\right), i=0,1, \ldots, L .
$$

Substituting (5) and (6) in (4), we get:

$$
m_{i}=\frac{\mu}{L\left((1+\mu)^{\frac{i}{L}}-(1+\mu)^{\frac{i-1}{L}}\right)}, i=1, \ldots, L .
$$

\subsection{Design of PLCSQ}

The first-degree spline function defined by (3) is used in design process of the PLCSQ. We consider the PLCSQ with support region defined by $\left[-x_{\max }, x_{\max }\right]$, that is divided into $2 L$ segments, where $N$ representation levels are placed. Each segment is further partitioned into uniform cells, where the step size, i.e. the uniform cell width, may differ from one segment to another, but the number of cells is equal in each segment and amounts to $n=N / 2 L$. As the symmetry condition holds (PLCSQ is symmetrical with respect to zero), both positive and negative half of the support region consists of $L$ segments. This allows us to consider only the positive half of the support region.

The width of cells within the $i$-th segment, denoted by $\Delta_{i}$, is given by [1]:

$$
\Delta_{i}=\frac{\Delta(N)}{c_{\mathrm{PLC}}^{\prime}\left(x, \sigma_{\mathrm{ref}}\right)}=\frac{\Delta(N)}{m_{i}}, i=1, \ldots, L,
$$

where

$$
\Delta(N)=\frac{2 x_{\max }}{N}
$$

Substituting (7) and (9) in (8), we derive:

$$
\Delta_{i}=\frac{2 L x_{\max }}{\mu N}\left((1+\mu)^{\frac{i}{L}}-(1+\mu)^{\frac{i-1}{L}}\right), i=1, \ldots, L .
$$

The decision thresholds of the $j$-th cell within the $i$-th segment, $x_{i j}$, can be determined as [1], [8]:

$$
\begin{aligned}
& c_{\mathrm{PLC}}\left(x_{i, j}, \sigma_{\mathrm{ref}}\right)=\frac{i-1}{L} x_{\mathrm{max}}+j \Delta(N) \Rightarrow \\
& x_{i, j}=c_{\mathrm{PLC}}^{-1}\left(\frac{i-1}{L} x_{\max }+j \Delta(N), \sigma_{\mathrm{ref}}\right)=x_{i-1}+j \Delta_{i}
\end{aligned}
$$

while the $j$-th representative level within the $i$-th cell, $y_{i, j}$, can be determined as [1], [8]: 


$$
\begin{aligned}
& c_{\mathrm{PLC}}\left(y_{i, j}, \sigma_{\mathrm{ref}}\right)=\frac{i-1}{L} x_{\max }+\frac{(2 j-1)}{2} \Delta(N) \Rightarrow \\
& y_{i, j}=c_{\mathrm{PLC}}^{-1}\left(\frac{i-1}{L} x_{\max }+\frac{(2 j-1)}{2} \Delta(N), \sigma_{\mathrm{ref}}\right)=x_{i-1}+\frac{(2 j-1)}{2} \Delta_{i}
\end{aligned}
$$

for $i=1, \ldots, L$ and $j=1, \ldots, n$. Obviously, the decision thresholds and the representative levels in the positive part are the reflection of the ones in the negative part of the support region.

The performance of the PLCSQ is characterized by the bit rate and MSE distortion [1], [2]. The bit rate is specified by $R=\log _{2} N=\log _{2}(2 \cdot L \cdot n)$ bits/sample. As in encoding process one bit is used for sign (positive or negative half of quantizer support region), the $i$-th segment where the input sample value falls is encoded with the natural binary code using $\log _{2} L$ bits (there are $L$ segments in one half of the support region) and the representative level to which the sample value is quantized is also encoded with natural binary code using $\log _{2} n$ bits (there are $n$ representative levels in one segment). MSE distortion is a measure of the error incurred during quantization [1], [2]. Typically, the total MSE distortion incorporates two components, the granular distortion $D_{\mathrm{g}}$ and overload distortion $D_{\mathrm{o}}$. The granular distortion in case when PLCSQ is designed for Laplacian PDF of variance $\sigma_{\text {ref }}^{2}$ and is applied to quantize another Laplacian PDF of variance $\sigma^{2}$ can be estimated as [1], [2]:

$$
D_{\mathrm{g}}=2 \sum_{i=1}^{L} \frac{\Delta_{i}^{2}}{12} \int_{x_{i-1}}^{x_{i}} p(x, \sigma) \mathrm{d} x
$$

while the overload distortion is evaluated as [1], [2]:

$$
D_{\mathrm{o}}=2 \int_{x_{\max }}^{\infty}\left(x-y_{L, n}\right)^{2} p(x, \sigma) \mathrm{d} x
$$

where $y_{L, n}$ is the $n$-th representative level within the $L$-th segment of the PLCSQ designed for $p\left(x, \sigma_{\mathrm{ref}}\right)$, as defined by (12). Substituting (1), (6) and (10) in (13) as well as (1) and (12) in (14), we derive the following expressions:

$$
\begin{aligned}
& D_{\mathrm{g}}=\frac{L^{2} x_{\max }^{2}}{3 N^{2} \mu^{2}} \sum_{i=1}^{L}(1+\mu)^{\frac{2(i-1)}{L}}\left((1+\mu)^{\frac{1}{L}}-1\right)^{2} \\
& \times \exp \left(-\frac{\sqrt{2} x_{\max }}{\mu \sigma}\left((1+\mu)^{\frac{i-1}{L}}-1\right)\right) \\
& \times\left(1-\exp \left(-\frac{\sqrt{2} x_{\max }}{\mu \sigma}\left((1+\mu)^{\frac{i}{L}}-(1+\mu)^{\frac{i-1}{L}}\right)\right),\right. \\
& D_{\mathrm{o}}=\exp \left(-\frac{\sqrt{2} x_{\max }}{\sigma}\right) \\
& \times\left(\left(\frac{(1+\mu) x_{\max } L\left(1-(1+\mu)^{-\frac{1}{L}}\right)}{\mu N}+\frac{\sigma}{\sqrt{2}}\right)+\frac{\sigma^{2}}{2}\right) \text {. }
\end{aligned}
$$

By using (15) and (16), the SQNR can be determined, which is a more indicative parameter for quantizer performance estimation [1], [2]:

$$
\mathrm{SQNR}=10 \log _{10}\left(\frac{\sigma^{2}}{D_{\mathrm{g}}+D_{\mathrm{o}}}\right) .
$$

Clearly, for $\sigma^{2}=\sigma_{\text {ref }}^{2}$ (i.e. for the variance matched case), one can estimate the performance of the described PLCSQ. According to (15)-(17), we can observe that $x_{\max }$ and $\mu$ are the critical parameters in our design. Typically, they are selected to satisfy the cost function (i.e. the minimal MSE distortion).

\subsection{Special Case of the Considered PLCSQ- G.711 Quantizer}

By adopting $\mu=\mu^{\mathrm{G} .711}=255$ and $N=256(L=8$ and $n=16$ ), the observed PLCSQ becomes equivalent to the one implemented in G.711 Recommendation [11], widely known as G.711 quantizer. An important characteristic of this quantizer is that it deals with unequal segments and each consecutive segment is twice as large as the previous. This property is not valid for described PLCSQ when $\mu \neq \mu^{\mathrm{G} .711}$. Moreover, as the optimized value of the upper support region threshold, $x_{\max }$ G.711, is not specified by G.711 recommendation, it is determined as in [21]:

$$
x_{\max }^{G .711}=\sigma_{\text {ref }} \frac{1}{\sqrt{2}} \log \left(\frac{3 \mu^{G .711} N^{2}}{\log ^{2}\left(\mu^{G .711}+1\right)}\right) .
$$

\section{Forward Adaptive DMSQ}

Forward adaptation is commonly used technique for implemention of scalar quantization models in non-stationary signals processing [6-8, 13]. It conducts frame-byframe analysis and processing, where frame denotes the finite length group of consecutive samples that can be considered as quasistationary. In this section, we propose a novel forward adaptive $N$-levels DMSQ incorporating two forward adaptive $N$-levels PLCSQs; one of them being G.711 quantizer (PLCSQs are described in the previous section). Using the similar terminology as in $[6-8,12]$, an $N$-levels PLCSQ having smaller support region is denoted here as the restricted quantizer and it is intended to quantize the frames whose amplitude content falls into its support region. Due to this fact, the restricted quantizer introduces only the granular component of the distortion (see (15)), while the overload one (see (16)) is avoided. Hence, the proper selection of the support region of the restricted quantizer within DMSQ is of great importance, and special attention is devoted to this (see Sec. 3.1). On the other hand, an $N$-levels PLCSQ having a wider support region (i.e. G.711 quantizer) with exactly specified support region threshold (eq. (18)) as well as compression factor value $(\mu=255)$, is denoted as the unrestricted quantizer and it is intended for the remaining signal frames. Using 
the forward adaptation, we are able to adjust the codebooks of employed PLCSQs to the input signal variations. In particular, before quantization is performed, parameters are extracted from the frame (e.g. frame variance) and used to scale the fixed (initial) sets of the representative levels and the decision thresholds of the respective quantizers. Obviously, defining the fixed codebooks is very important task and deserves more attention. In the following, we will explain the design of fixed DMSQ followed by a detailed description of the forward adaptive scheme.

\subsection{Design of Fixed DMSQ}

During the design process we use the memoryless Laplacian source with zero-mean and unit variance, i.e. $p\left(x, \sigma_{\text {ref }}=1\right)$, which is the most commonly used assumption in quantizer design [1]. The design goal is to enable a more frequent choice of the restricted quantizer in order to exploit the full benefit of dual-mode quantization. As the structure of DMSQ components is described in detail in the previous section, here we focus on the optimization of the parameters affecting the overall performance. Specifically, given PDF, an $N$-levels DMSQ composed of $N$-levels restricted and $N$-levels unrestricted quanizers is designed such that minimal MSE distortion (maximal SQNR) is provided. The total MSE distortion, $D_{\mathrm{t}}$, of the fixed DMSQ can be calculated as $[6-8,12]$ :

$$
D_{\mathrm{t}}=P^{\mathrm{r}} D^{\mathrm{r}}+\left(1-P^{\mathrm{r}}\right) D^{\text {ur }}
$$

where $D^{\mathrm{r}}=D^{\mathrm{r}}\left(\sigma_{\text {ref }}\right)$ denotes the granular distortion of the restricted quantizer designed for $p\left(x, \sigma_{\mathrm{ref}}=1\right)$ (see (15)), $D^{\mathrm{ur}}=D^{\mathrm{ur}}\left(\sigma_{\mathrm{ref}}\right)$ denotes the total distortion of unrestricted quantizer designed for $p\left(x, \sigma_{\text {ref }}=1\right)$ (the sum of granular (15) and overload (16) distortions), while $P^{\mathrm{r}}=P^{\mathrm{r}}\left(\sigma_{\text {ref }}\right)$ refers to the probability of selecting the restricted quantizer having the support region $\left[-x_{\max }^{\mathrm{r}}, x_{\max }{ }^{\mathrm{r}}\right][6-8,12]$ :

$$
P^{\mathrm{r}}=\left(2 \int_{0}^{x_{\max }^{\mathrm{r}}} p\left(x, \sigma_{\text {ref }}=1\right) \mathrm{d} x\right)^{M}=\left(1-\exp \left(-\sqrt{2} x_{\max }^{\mathrm{r}}\right)\right)^{M}
$$

where $M$ is the frame length. Clearly, $1-P^{\mathrm{r}}$ in (19) stands for the probability that unrestricted quantizer is selected.

SQNR of DMSQ is given by [12]:

$$
\mathrm{SQNR}=10 \log _{10}\left(\frac{1}{P^{\mathrm{r}} D^{\mathrm{r}}+\left(1-P^{\mathrm{r}}\right) D^{\mathrm{ur}}}\right) .
$$

Let us further define the bit rate. To represent each sample from the particular frame we use fixed-length codewords of $\log _{2} N$ bits (we deal with restricted and unrestricted quantizers both having equal $N$ ), while the information about the selected quantizer (restricted or unrestricted) is represented by one bit per each frame, which gives [12]:

$$
R^{\mathrm{fix}}=\log _{2} N+\frac{1}{M}
$$

Recall that unrestricted (G.711) quantizer is completely defined $\left(\mu^{\mathrm{G} .711}\right.$ and $x_{\max }$ G.711 are provided), therefore according to $(19)-(21)$, we can conclude that the performance of the $N$-level DMSQ is strongly dependent on $x_{\max }{ }^{\mathrm{r}}$ and $\mu^{\mathrm{r}}$ denoting the parameters of the $N$-levels restricted quantizer. Accordingly, we optimize these parameters from:

$$
\begin{gathered}
\frac{\partial D_{\mathrm{t}}}{\partial x_{\max }^{\mathrm{r}}}=0 \Rightarrow x_{\max }^{\mathrm{r}}=x_{\max }^{\mathrm{r}, \mathrm{opt}}, \\
\frac{\partial D_{\mathrm{t}}}{\partial \mu^{\mathrm{r}}}=0 \Rightarrow \mu^{\mathrm{r}}=\mu^{\mathrm{r}, \mathrm{opt}} .
\end{gathered}
$$

Hence, optimization of the parameters of the restricted quantizer (i.e. its proper design) within DMSQ can be viewed as one of the contributions presented in this paper. Furthermore, we analyze the problem of selecting the suitable frame length $M$, which affects both $P^{\mathrm{r}}$ (i.e. SQNR) and $R^{\text {fix }}$. We propose its selection according to the maximal gain in SQNR achieved versus G.711 quantizer.

In Fig. 1, we illustrate SQNR dependence on $M$ for three versions of the proposed $N=256$-levels DMSQ optimized in terms of minimal MSE distortion, i.e. the cases where the restricted quantizer is designed with $c_{\mathrm{PLC}}\left(x, \sigma_{\mathrm{ref}}\right)$ composed of $L=2, L=4$ and $L=8$ line segments. In a given figure, for comparison purposes, we also provide SQNR curve for $N=256$ levels-DMSQ baseline reported in [12], which serves as the upper bound of performance due to the fact that PLCSQs always provide lower SQNR in comparison to scalar compandors based on nonlinear compression function [22], [23]. Note that the performance in terms of SQNR of the proposed DMSQ with the restricted quantizer designed by $c_{\mathrm{PLC}}\left(x, \sigma_{\text {ref }}\right)$ using higher number of segments $(L=8)$ converges to the upper bound given in [12].

In order to fairly estimate the gain in SQNR of the proposed model versus the single G.711 quantizer, it is preferable to determine the SQNR values achieved for the same bit rate, i.e. for the bit rate of 8 bit/sample. Recall that our fixed DMSQ has increased bit rate for $1 / M$ bits when compared to the baseline (see (22)). Therefore, the estimated SQNR value at the bit rate of $8 \mathrm{bit} / \mathrm{sample}$ can be determined as [12]:

$$
\mathrm{SQNR}_{\mathrm{es}}[\mathrm{dB}]=\mathrm{SQNR}[\mathrm{dB}]-\frac{6}{M}[\mathrm{~dB}]
$$

since in high-rate scalar quantization with one extra bit an improvement of $6 \mathrm{~dB}$ in SQNR is provided [1].

The dependence of $\mathrm{SQNR}_{\mathrm{es}}$ on $M$ is depicted in Fig. 2, from which we can easily determine the optimal frame lengths for fixed DMSQ, which depends on the design of the restricted quantizer. In particular, when the restricted quantizer uses $c_{\mathrm{PLC}}\left(x, \sigma_{\text {ref }}\right)$ with $L=2, L=4$ and $L=8$ segments, the optimal frame lengths for DMSQ are $M=5\left(x_{\max }^{\text {ropt }}=2.92, \mu^{\mathrm{r}, \mathrm{opt}}=2.27\right), M=6\left(x_{\max }{ }^{\mathrm{r}, \mathrm{opt}}=3.17\right.$, $\left.\mu^{\text {ropt }}=2.87\right)$ and $M=7\left(x_{\max }^{\text {ropt }}=3.32, \mu^{\text {r,opt }}=3.21\right)$, respectively, showing the gain in SQNR of over $5 \mathrm{~dB}$ in compari- 
son to the G.711 quantizer. For such established parameters, the restricted quantizer is chosen with probabilities $P^{\mathrm{r}}(L=2)=0.922, P^{\mathrm{r}}(L=4)=0.934, P^{\mathrm{r}}(L=8)=0.938$, which is favorable in case of dual-mode scalar quantization.

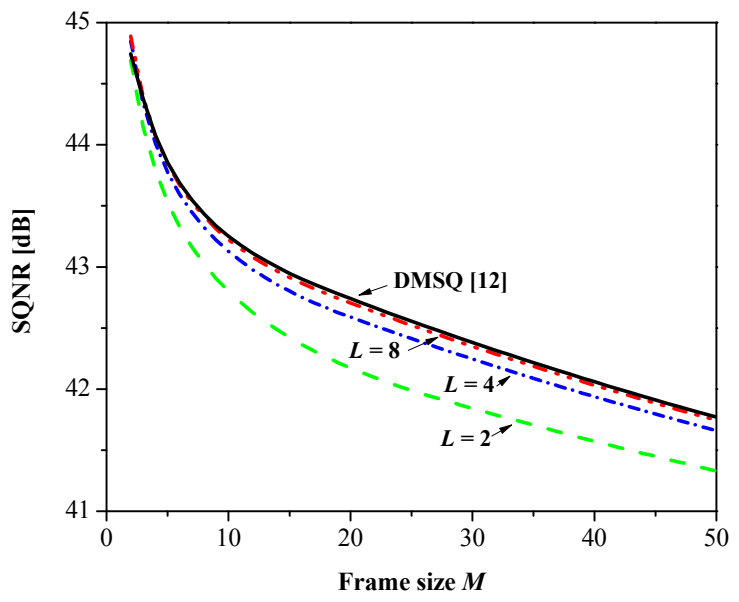

Fig. 1. SQNR versus $M$ for different implementations of the proposed $N=256$ levels-DMSQ and baseline in [12], in the case of optimally chosen $x_{\max }{ }^{\mathrm{r}}$ and $\mu^{\mathrm{r}}$.

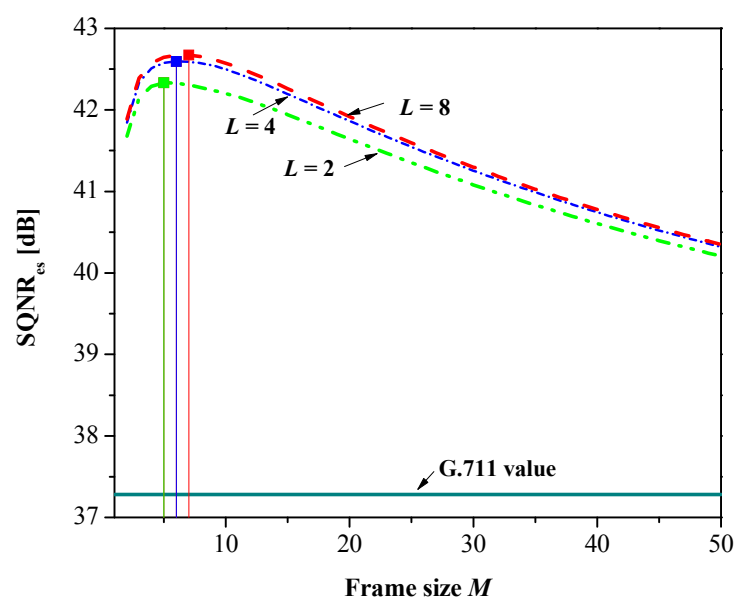

Fig. 2. SQNR $\mathrm{ex}_{\mathrm{ex}}$ versus $M$ for different implementations of the proposed $N=256$ levels-DMSQ.

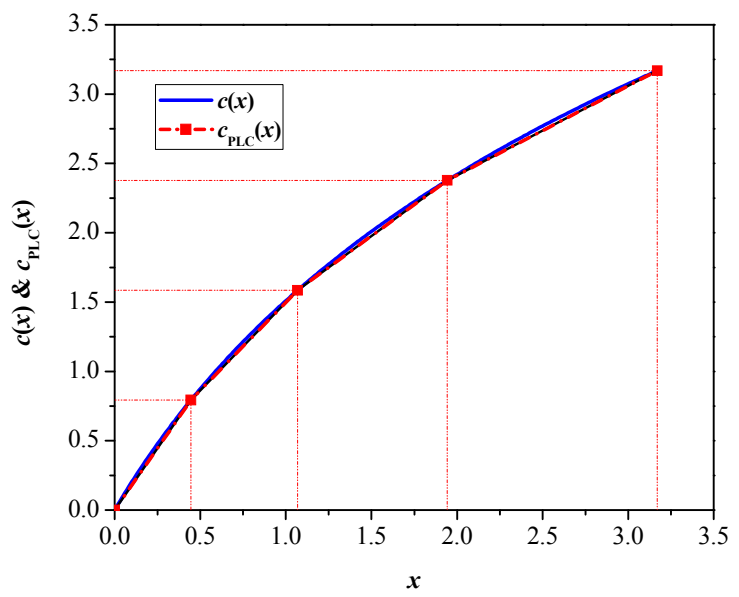

Fig. 3. First-degree spline approximation with $L=4$ segments used in design of $N=256$-levels restricted quantizer $\left(x_{\max }^{\mathrm{r}}=3.17, \mu^{\mathrm{r}}=2.87\right)$
For illustrative purposes, in Fig. 3 we show $c_{\text {PLC }}\left(x, \sigma_{\text {ref }}\right)$ with $L=4$ line segments in the positive part of the characteristic, used in the design of the $N=256$ levelsrestricted quantizer, for optimally selected parameters $\left(x_{\max }^{\mathrm{r}, \mathrm{opt}}=3.17, \mu^{\mathrm{r}, \mathrm{opt}}=2.87\right)$. In particular, DMSQ composed of such restricted quantizer and unrestricted G.711 quantizer is used in further analysis.

\subsection{The Proposed Scheme}

In forward adaptation technique, beside quantizer output codewords, there is a need to transmit some additional parameters to the decoder as side information [1], [13]. Hence, the side information can be a critical parameter, as it can have a large impact on the overall bit rate. As it has been demonstrated, the optimal frame length for our fixed DMSQ is very small $(M=6)$, which means that in the forward adaptive scheme side information has to be sent very often, which is unfavorable. On the other hand, the method applied in the solutions developed so far [6-8] that use longer frames (typically $M=41$ and $M=51$ ) is obviously not adequate enough. Therefore, we here propose the configuration with frame/subframe logic, where adaptation to variance is performed at the frame level and adaptation to the maximal amplitude is performed at the subframe level. Therefore, the frames are used only for adaptation, while subframes are used for selection of the appropriate restricted/unrestricted quantizer. The goal is evident: by division of the frame into subframes a large overhead can be reduced; furthermore, the more frequent selection of the restricted quantizer is assured and accordingly the increasing overall performance is expected.

The block diagram of the proposed scheme with encoder and decoder parts is depicted in Fig. 4(a) and Fig. 4(b), respectively. As Fig. 4(a) shows, the encoder contains blocks for buffering, the variance estimation, the variance quantization, subframe forming, the estimation of maximal absolute amplitude of the subframe and adaptive restricted and unrestricted quantizers, both having $N$-levels. The buffer is used to store $M_{0}$ consecutive samples (i.e. one frame), $x_{j}[n], n=1, \ldots, M_{0}$, where $j$ denotes the frame index. For a current frame, the variance is estimated in the variance estimation block as [6], [7]:

$$
\sigma_{j}^{2}=\frac{1}{M_{0}} \sum_{n=1}^{M_{0}} x_{j}^{2}[n]
$$

This parameter is very important as it is used to scale the parameters of the adaptive restricted and unrestricted quantizers composing DMSQ. Hence, for each signal frame, information about the variance has to be available at the decoder side, which implies its quantization. In particular, the variance of the $j$-th frame is firstly transformed into log-domain, $\alpha_{j}[\mathrm{~dB}]=10 \log _{10}\left(\sigma_{j}^{2} / \sigma_{\text {ref }}^{2}\right)$, and then quantized using the log-uniform quantizer $\left(\mathrm{Q}_{\mathrm{LU}}\right)$ having $S$ quantization points, as it can provide better performance in regard to other quantizer models (e.g. uniform quantizer) [6-8]. We define $Q_{L U}$ as [6]: 


$$
\alpha_{j} \in \alpha_{k}[\mathrm{~dB}] \mid \alpha_{k}[\mathrm{~dB}]=\alpha_{\min }+(2 k-1) \frac{B}{2 S}, k=1, \ldots, S
$$

where $B[\mathrm{~dB}]=\alpha_{\max }[\mathrm{dB}]-\alpha_{\min }[\mathrm{dB}]$ is the dynamic range. The chosen level of the $\mathrm{Q}_{\mathrm{LU}}$ (i.e. the quantized frame variance) is encoded using fixed-length codewords of $R_{S}=\log _{2} S$ bits (index $K$ ).

Based on the quantized frame variance, the set of decision thresholds $\mathbf{T}^{\mathrm{r}}(g)$ and the set of representative levels $\mathbf{Y}^{\mathrm{r}}(g)$ of the adaptive restricted quantizer, as well as the adaptive unrestricted quantizer, respectively denoted as $\mathbf{T}^{\mathrm{ur}}(g)$ and $\mathbf{Y}^{\mathrm{ur}}(g)$, are obtained as follows:

$$
\begin{aligned}
& \mathbf{T}^{\mathrm{r}}(g)=g \cdot \mathbf{T}^{\mathrm{r}}\left(\sigma_{\text {ref }}\right), \mathbf{Y}^{\mathrm{r}}(g)=g \cdot \mathbf{Y}^{\mathrm{r}}\left(\sigma_{\text {ref }}\right), \\
& \mathbf{T}^{\mathrm{ur}}(g)=g \cdot \mathbf{T}^{\mathrm{ur}}\left(\sigma_{\text {ref }}\right), \mathbf{Y}^{\mathrm{ur}}(g)=g \cdot \mathbf{Y}\left(\sigma_{\text {ref }}\right)
\end{aligned}
$$

where $\mathbf{T}^{\mathrm{r}}\left(\sigma_{\text {ref }}\right), \mathbf{Y}^{\mathrm{r}}\left(\sigma_{\text {ref }}\right), \mathbf{Y}^{\mathrm{ur}}\left(\sigma_{\text {ref }}\right), \mathbf{T}^{\mathrm{ur}}\left(\sigma_{\text {ref }}\right)$ stand for the sets of the respective quantizers designed for reference variance $\left(\sigma_{\text {ref }}^{2}=1\right)$, while $g$ is the scaling factor defined as:

$$
g=\frac{10^{\alpha_{k} / 20}}{\sigma_{\text {ref }}} .
$$

After the adaptation to the variance is performed, the adaptation to the maximal amplitude is applied in the next step, providing a basis for selection of the adaptive re- stricted or unrestricted quantizer. The $j$-th frame of $M_{0}$ samples stored in the buffer is further divided into the subframes of $M$ samples (subframe forming block in Fig. 4(a)). For each subframe $\left(x_{j, l}[n], l=1, \ldots, M_{0} / M\right.$, $n=1, \ldots, M$, denote the $l$-th subframe within the $j$-th frame), the maximal absolute amplitude $\left|x_{j, l}[n]\right|_{\max }$ need to be estimated and further compared with the properly chosen threshold value $x_{\mathrm{t}}$ in order to make the decision which adaptive quantizer has to be used. Similarly as in [6-8], we set $x_{\mathrm{t}}=x_{\max }^{\mathrm{r}}(g)$ (upper support region threshold of the adaptive restricted quantizer). As with the restricted quantizer we want to introduce only the granular distortion, for its selection the inequality $\left|x_{j, l}[n]\right|_{\max } \leq x_{\max }{ }^{\mathrm{r}}(g)$ must hold, otherwise the unrestricted quantizer is used. Information about the selected quantizer is also sent to the decoder and requires one bit per each subframe (index $J$ ). Finally, the samples from the current subframe are individually passed through the selected adaptive quantizer and encoded using the fixed-length codewords composed of $\log _{2} N$ bits (index $I$ ).

According to Fig. 4(a), three indexes $I, J$ and $K$ have to be transmitted to the decoder, arriving at the bit rate for the forward adaptive DMSQ:

$$
R^{\mathrm{FA}}=\log _{2} N+\frac{1}{M}+\frac{\log _{2} S}{M_{0}} .
$$

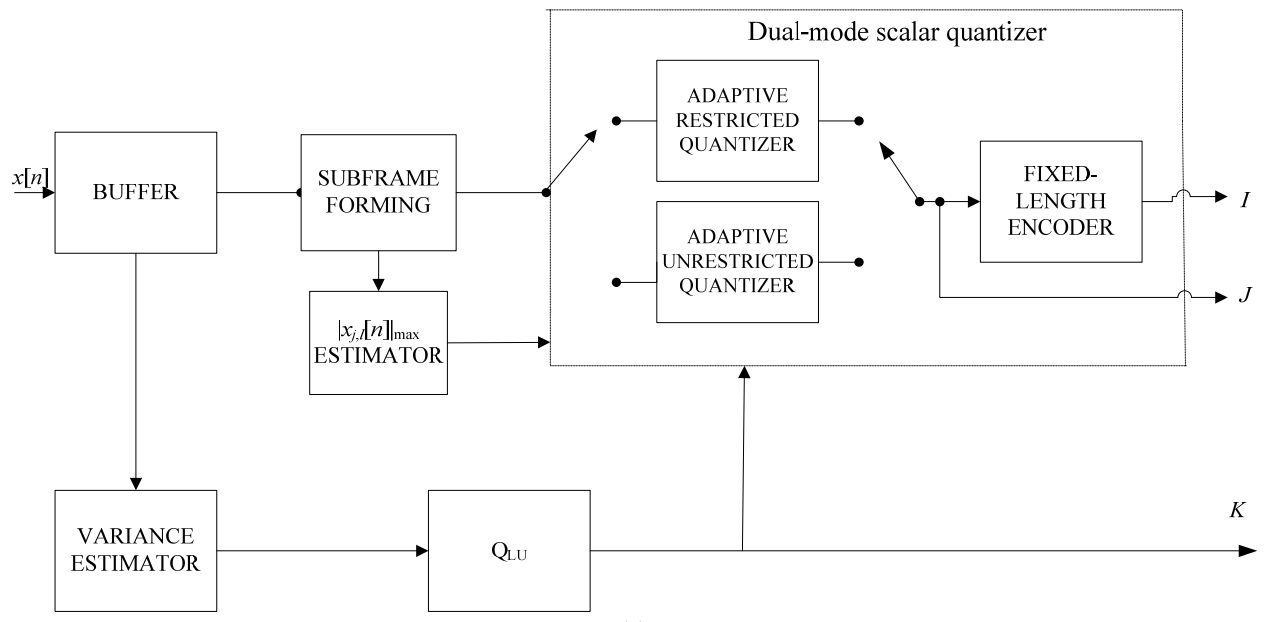

(a)

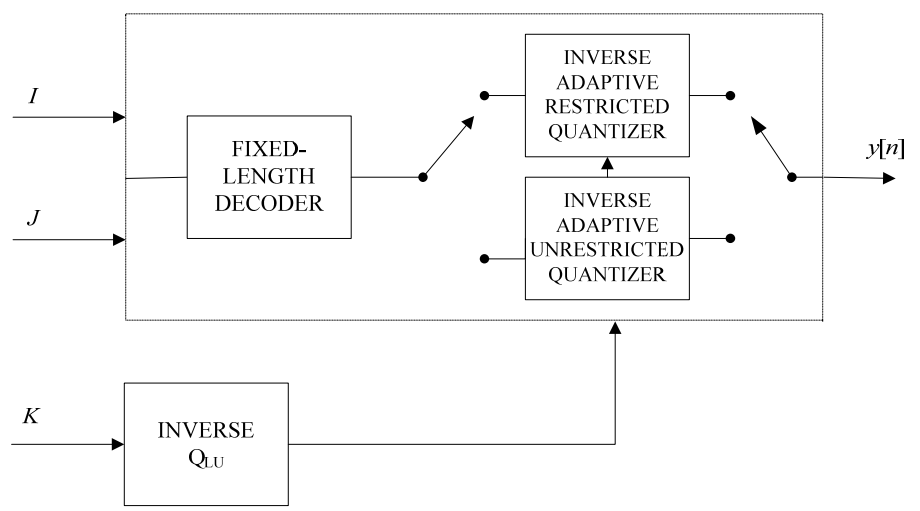

(b)

Fig. 4. Forward adaptive coding scheme: a) encoder, b) decoder. 
Decoder is shown in Fig. 4(b), which reconstructs the signal frames with the length of $M_{0}$ samples using these three indexes $(I, J$ and $K)$.

Eventually, the performance of the forward adaptive $N$-level DMSQ can be estimated using the following set of equations:

$$
\begin{gathered}
\operatorname{SQNR}(g, \sigma)=10 \log _{10}\left(\frac{\sigma^{2}}{D_{\mathrm{t}}(g, \sigma)}\right) \\
D_{\mathrm{t}}(g, \sigma)=P^{\mathrm{r}}(g, \sigma) D^{\mathrm{r}}(g, \sigma)+\left(1-P^{\mathrm{r}}(g, \sigma)\right) D^{\mathrm{ur}}(g, \sigma) \\
P^{\mathrm{r}}(g, \sigma)=\left(1-\exp \left(-\sqrt{2} x_{\text {max }}^{\mathrm{r}}(g) / \sigma\right)\right)^{M} \\
x_{\text {max }}(g)=g \cdot x_{\text {max }}\left(\sigma_{\text {ref }}\right)
\end{gathered}
$$

\section{Results and Discussion}

In this section, the SQNR performance of the proposed forward adaptive DMSQ is theoretically analyzed, assuming that input signal follows the Laplacian PDF and its variance changes in a wide range $(B=[-20 \mathrm{~dB}, 20 \mathrm{~dB}])$. In addition, the theoretical analysis is supported by the experimental one using a real speech signal. In particular, we consider $N=256$-levels DMSQ that incorporates $N=256$ levels restricted quantizer $\left(c_{\mathrm{PLC}}(x)\right.$ is composed of $L=4$ line segments, $\left.x_{\max }^{\mathrm{r}}\left(\sigma_{\mathrm{ref}}\right)=3.17, \mu^{\mathrm{r}}=2.87\right)$ and $N=256$-levels unrestricted quantizer $\left(c_{\text {PLC }}(x)\right.$ is composed of $L=8$ segments, $\left.x_{\max }^{\mathrm{G} .711}\left(\sigma_{\text {ref }}\right)=10.11, \mu^{\mathrm{G} .711}=255\right)$ and subframe length is set to $M=6$.

As mentioned earlier, for the proposed forward adaptive DMSQ the side information is very important parameter affecting the overall bit rate and it is influenced by two parameters, $S$ and $M_{0}$ (see (31)). Therefore, the most suitable version of the proposed codec requires the optimized values of these parameters, which is not a trivial task. The importance of codec parameters optimization has been indicated in [24]. In this paper, given fixed $M_{0}$, we optimize $S$. In other words, we investigate the influence of number of levels of $\mathrm{Q}_{\mathrm{LU}}, S$, on the performance of the proposed DMSQ defined by the average SQNR [6]:

$$
\operatorname{SQNR}_{\mathrm{av}}=\frac{1}{m} \sum_{i=1}^{m} \operatorname{SQNR}\left(\sigma_{i}\right)
$$

where $m$ is the number of particular variances taken into account in the established range $|B|=40 \mathrm{~dB}$. Selection of the optimal $S$ is performed using a special criterion:

$$
\frac{\operatorname{SQNR}_{\mathrm{av}}\left(N, M_{0}, S\right)-\mathrm{SQNR}_{\mathrm{av}}\left(N, M_{0}, S / 2\right)}{R^{\mathrm{FA}}\left(N, M_{0}, S\right)-R^{\mathrm{FA}}\left(N, M_{0}, S / 2\right)} \geq 6 \frac{\mathrm{dB}}{\mathrm{bit}}
$$

which defines that there is no point to further increase the number of levels $S$ if the unit increase of the number of bits per sample does not result in the average SQNR increase of $6 \mathrm{~dB}$ or more.

\begin{tabular}{|c|c|c|}
\hline $\boldsymbol{S}\left(\boldsymbol{R}_{\mathbf{s}}\right)$ & $\mathbf{S Q N R}_{\mathbf{a v}}[\mathrm{dB}]$ & $\mathbf{R}^{\mathbf{F A}}[\mathrm{b} / \mathrm{s}]$ \\
\hline $2(1)$ & 36.9920 & 8.1708 \\
\hline $4(2)$ & 41.8159 & 8.1750 \\
\hline $8(3)$ & 43.2025 & 8.1792 \\
\hline $16(4)$ & 43.4956 & 8.1833 \\
\hline $\mathbf{3 2}(\mathbf{5})$ & $\mathbf{4 3 . 5 6 7 0}$ & $\mathbf{8 . 1 8 7 5}$ \\
\hline $64(6)$ & 43.5848 & 8.1917 \\
\hline $128(7)$ & 43.5893 & 8.1958 \\
\hline $256(8)$ & 43.5904 & 8.2000 \\
\hline
\end{tabular}

Tab. 1. The values of average SQNR and bit rate for $N=256$-levels forward adaptive DMSQ achieved for different $S, M_{0}=240$ samples.

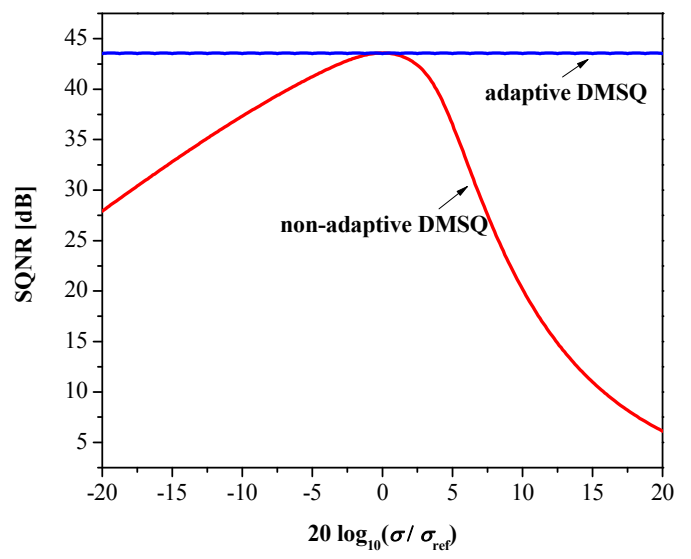

Fig. 5. Theory: SQNR of the forward adaptive $\left(M_{0}=240\right.$, $M=6, S=32$-levels $\left.\mathrm{Q}_{\mathrm{Lu}}, R^{\mathrm{FA}}=8.1875 \mathrm{bit} / \mathrm{sample}\right)$ and non-adaptive $\quad N=256 \quad$ levels-DMSQ $\quad(M=6$, $R^{\text {fix }}=8.1667 \mathrm{bit} / \mathrm{sample}$ ) in the wide range of input signal variances $|B|=40 \mathrm{~dB}$.

Table 1 summarizes the achieved $\mathrm{SQNR}_{\mathrm{av}}$ values together with values of the bit rate for the forward adaptive DMSQ, when $S$ varies (i.e. $R_{\mathrm{S}}$ ranges from 1 to 8 bits) and $M_{0}=240$. By applying (37), it is revealed that the required optimal value is $S=32$ ( $R_{\mathrm{s}}=5$ bits), for a given frame size.

In Fig. 5, where SQNR is shown in a wide range of the input signal variances, we provide the results for forward adaptive $\left(M_{0}=240, S=32\right.$-levels $\left.\mathrm{Q}_{\mathrm{LU}}\right)$ and nonadaptive (designed for $\sigma_{\text {ref }}^{2}=1$ ) DMSQs with $N=256$ levels. The advantage is clearly visible, as forward adaptive version retains almost constant SQNR in the entire variance range and gives more than $14 \mathrm{~dB}$ higher average SQNR in comparison to the non-adaptive one. In this paper, our goal is also to examine the benefit of the proposed forward adaptive $N=256$-levels DMSQ $\left(R^{\mathrm{FA}}=8.1875 \mathrm{bit} / \mathrm{sample}\right)$ with respect to different baselines, as shown in Fig. 6. It is easy to ascertain that our quantizer completely satisfies G.712 Recommendation [25], defining the minimum SQNR value (in the wide variance range of almost $40 \mathrm{~dB}$ ) in systems for high-quality speech transmission. Moreover, it attains much higher and constant SQNR values in comparison to the ones specified by G.711 Recommendation [11], with the gain in maximal SQNR of approximatelly $6 \mathrm{~dB}$; the gain also increases in the rest of the considered range. If we compare the attained average SQNR values, then the gain of $11.3 \mathrm{~dB}$ can be observed $\left(\mathrm{SQNR}_{\mathrm{av}}^{\mathrm{G} .711}=32.29 \mathrm{~dB}[1]\right)$. 


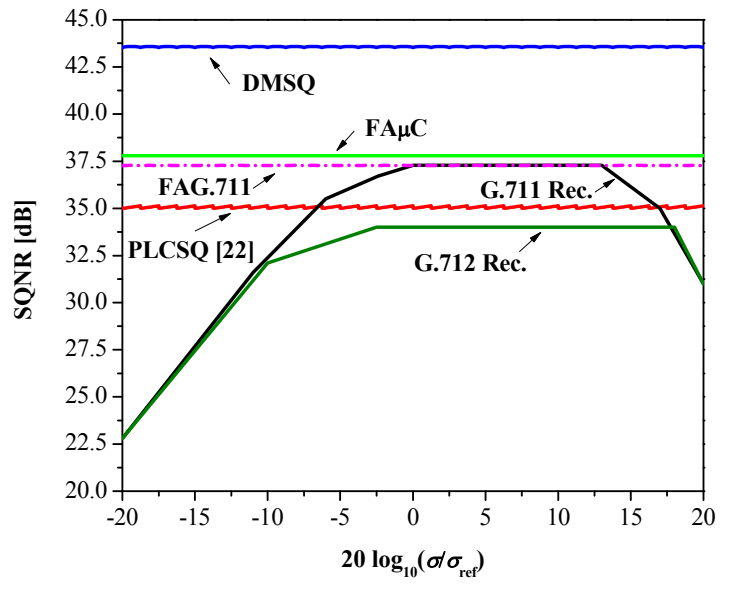

Fig. 6. Theory: Performance in terms of SQNR of the proposed forward adaptive $N=256$-levels DMSQ $\left(R^{\mathrm{FA}}=8.1875 \mathrm{bit} / \mathrm{sample}\right)$ and different baselines in the wide range of input signal variances $|B|=40 \mathrm{~dB}$, $S=32$-levels $\mathrm{Q}_{\mathrm{Lu}}, M_{0}=240$.

The superiority of our solution can also be perceived with respect to other baselines based on the forward adaptive technique. As baseline approaches scheme, we use G.711 quantizer (FAG.711), $N=256$-levels $\mu$-law compandor $(\mu=255)$ described in [12] (FA $\mu \mathrm{C})$ and the forward adaptive $N=256$-levels PLCSQ from [22], which is based on approximation of optimal compression function with the first-degree spline functions. We report the gains in average SQNR of $6 \mathrm{~dB}$ when compared to FAG.711, $5.77 \mathrm{~dB}$ in regard to $\mathrm{FA} \mu \mathrm{C}$ and $8.5 \mathrm{~dB}$ in regard to the solution from [22], achieved at the cost of slightly increased bit rate of $0.1667 \mathrm{bit} / \mathrm{sample}$ (information about the selected quantizer, one-bit per subframe).

Furthermore, in Fig. 7, we provide the SQNR characteristic for forward adaptive $N=128$-levels DMSQ (consisted of $N=128$-levels restricted and $N=128$-levels unrestricted quantizers). Note that the optimized parameters of the restricted quantizer as well as the optimal subframe length are determined in a similar way as it was done for $N=256-$ levels DMSQ. The advantage of the proposed

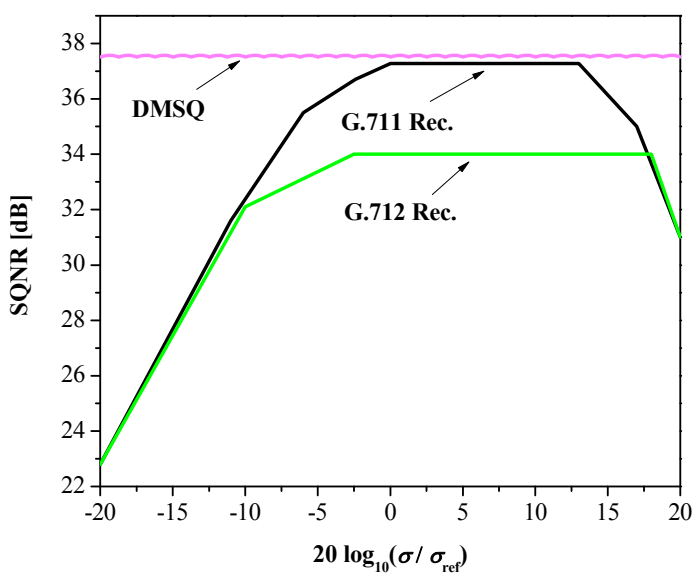

Fig. 7. Theory: SQNR of forward adaptive DMSQ with $N=128$ levels $\left(R^{\mathrm{FA}}=7.1875 \mathrm{bit} / \mathrm{sample}\right)$ in the wide range of input signal variances $|B|=40 \mathrm{~dB}, M_{0}=240$, $M=6, S=32$-levels $\mathrm{Q}_{\mathrm{Lu}}$.

\begin{tabular}{|c|c|c|c|c|c|}
\hline & $\begin{array}{c}\text { G.711 } \\
\boldsymbol{N}=\mathbf{2 5 6}\end{array}$ & $\begin{array}{c}\text { FA } \boldsymbol{\mu C} \\
\boldsymbol{N}=\mathbf{2 5 6}\end{array}$ & $\begin{array}{c}\text { PLCSQ }^{[22]} \\
\boldsymbol{N}=\mathbf{2 5 6}\end{array}$ & $\begin{array}{c}\text { DMSQ } \\
\boldsymbol{N}=\mathbf{1 2 8}\end{array}$ & $\begin{array}{c}\text { DMSQ } \\
\boldsymbol{N}=\mathbf{2 5 6}\end{array}$ \\
\hline $\begin{array}{c}\mathbf{S Q N R}_{\text {seg }} \\
{[\mathbf{d B}]}\end{array}$ & 33.3225 & 37.8106 & 34.8551 & 36.9717 & 42.9759 \\
\hline $\begin{array}{c}\mathbf{R}^{\mathbf{F A}} \\
{[\mathbf{b} / \mathbf{s}]}\end{array}$ & 8 & 8.0208 & 8.0208 & 7.1875 & 8.1875 \\
\hline
\end{tabular}

Tab. 2. Experiment: $\mathrm{SQNR}_{\text {seg }}$ and bit rate achieved for available speech signal, $S=32$-levels $\mathrm{Q}_{\mathrm{LU}}, M_{0}=240$ samples.
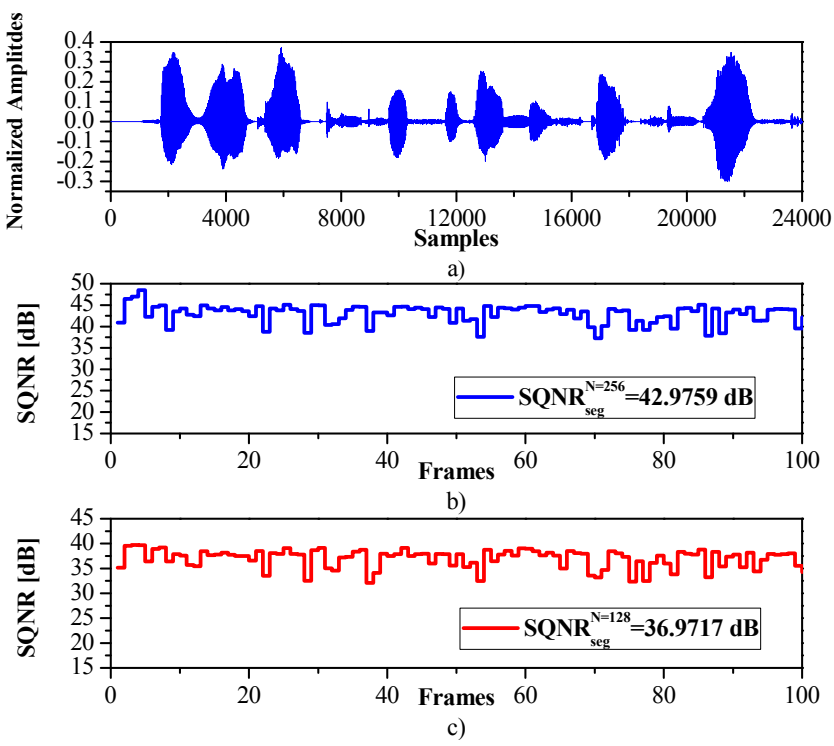

Fig. 8. Experiment: SQNR over the frames containing 240 samples for adaptive DMSQ with $N=128$ and $N=256$ levels, $M=6$ and $S=32$-levels $\mathrm{Q}_{\mathrm{LU}}$

codec $\left(R^{\mathrm{FA}}=7.1875 \mathrm{bit} / \mathrm{sample}\right)$ is obvious, since $5 \mathrm{~dB}$ gain in the average SQNR over G.711 quantizer is obtained along with $0.8125 \mathrm{bit} / \mathrm{sample}$ lower bit rate, at the same time completely satisfying G.712 Recommendation.

Finally, the proposed forward adaptive DMSQ is tested for speech coding, as it has been shown that speech is well modeled by Laplacian PDF [26]. Experiments are performed on a speech signal extracted from the TIMIT dataset [20], and sampled at the frequency of $8 \mathrm{kHz}$. The test speech contains 24000 samples. As a measure of performance the segmental SQNR is used, i.e. the SQNR is calculated over each speech frame and then averaged [2].

Experimental results are presented in Tab. 2 and Fig. 8, showing a good matching with the theoretical ones presented in Figs. 6 and 7.

\section{Summary and Conclusion}

In this paper, a full potential of the recently proposed dual-mode quantization technique versus conventional fixed-rate quantization has been ascertained. In particular, we have proposed DMSQ solution for processing signals with the Laplacian distribution, which employs two similar PLCSQs, the restricted and unrestricted ones, having an equal number of quantization levels and unequal support regions. Both PLCSQs have been designed using the first- 
degree spline function, while the key parameters of the restricted quantizers within DMSQ have been optimized in terms of the minimal MSE distortion. The implementation in the forward adaptive scheme has been done, where the frame/subframe method has been proposed. To analyze the performance of this model, the theoretical and the experimental analysis has been provided. Theoretical results have proved a high level of robustness in a wide range of input signal variances. For the same number of levels $(N=256)$, it has been shown that the proposed quantizer significantly outperforms G.711 quantizer, with the achieved gain in average SQNR of more than $11 \mathrm{~dB}$. Moreover, the performance of G.711 baseline has been overreached with only $N=128$ levels, where the gain in average SQNR of more than $5 \mathrm{~dB}$ along with $0.8215 \mathrm{bit} / \mathrm{sample}$ lower bit rate has been achieved. Since high-quality quantization can be achieved at lower bit rate in comparison to the G.711 codec which is embedded within the proposed solution, it makes the proposed model a good candidate for a wide range of applications dealing with Laplacian sources, e.g. for speech coding.

\section{Acknowledgments}

This work was supported in part by the Ministry of Education and Science of the Republic of Serbia, grant no. TR32035 and TR32051 within the Technological Development Program.

\section{References}

[1] JAYANT, N., NOLL, P. Digital Coding of Waveforms: Principles and Applications to Speech and Video. New Jersey: Prentice Hall, 1984. ISBN: 978-0132119139

[2] CHU, C. Speech Coding Algorithms. New Jersey: John Wiley \& Sons, 2005. ISBN: 9780471668879

[3] NA, S., NEUHOFF, D. L. Monotonicity of step sizes of MSEoptimal symmetric uniform scalar quantizers. IEEE Transactions on Information Theory, 2019, vol. 65, no. 3, p. 1782-1792. DOI: 10.1109/TIT.2018.2867182

[4] NA, S., NEUHOFF, D. L. On the convexity of the MSE distortion of symmetric uniform scalar quantization. IEEE Transactions on Information Theory, 2018, vol. 64, no. 4, p. 2626-2638. DOI: 10.1109/TIT.2017.2775615

[5] PERIC, Z., MOSIC, A., PANIC, S. Coding algorithm based on loss compression using scalar quantization switching technique and logarithmic companding. Journal of Information Science and Engineering, 2010, vol. 26, no. 3, p. 967-976.

[6] PERIC, Z., NIKOLIC, J. High-quality Laplacian source quantisation using a combination of restricted and unrestricted logarithmic quantisers. IET Signal Processing, 2012, vol. 6, no. 7, p. 633-640. DOI: 10.1049 /iet-spr.2011.0213

[7] NIKOLIC, J., PERIC, Z. An adaptive waveform coding algorithm and its application in speech coding. Digital Signal Processing, 2012, vol. 22, no. 1, p. 199-209. DOI: 10.1016/j.dsp.2011.09.001

[8] NIKOLIC, J., PERIC, Z., JOVANOVIC, A. Two forward adaptive dual-mode companding scalar quantizers for Gaussian source. Signal Processing, 2016, vol. 120, p. 129-140. DOI: 10.1016/j.sigpro.2015.08.016

[9] NA, S. On the support of fixed-rate minimum mean-squared error scalar quantizers for a Laplacian source. IEEE Transactions on Information Theory, 2004, vol. 50, no. 5, p. 937-944. DOI: 10.1109/TIT.2004.826686

[10] NA. S., NEUHOFF, D. L. On the support of MSE-optimal, fixedrate scalar quantizers. IEEE Transactions on Information Theory, 2001, vol. 47, no. 7, p. 2972-2982. DOI: 10.1109/18.959274

[11] INTERNATIONAL TELECOMMUNICATION UNION. ITU-T G.711: Pulse Code Modulation of Voice Frequencies, 1972.

[12] DENIC, B., PERIC, Z., DESPOTOVIC, V., et al. Dual-mode quasi-logarithmic quantizer with G.711 codec. Journal of Electrical Engineering, 2018, vol. 69, no. 1, p. 46-51. DOI: 10.1515/jee-2018-0006

[13] ORTEGA, A., VETTERLY, M. Adaptive scalar quantization without side information. IEEE Transactions on Image Processing, 1997, vol. 6, no. 5, p. 665-676. DOI: $10.1109 / 83.568924$

[14] DINCIC, M., PERIC, Z. Log-polar quantizer with the embedded ITU-T G.711 codec. Radioengineering, 2010, vol. 19, no. 4, p. 712-717. ISSN: $1210-2512$

[15] ZHAO, Z., LIU, H., FINGSCHEIDT, T. Enhancement of G.711coded speech providing quality higher than uncoded. In Proceedings of the 13th ITG-Fb. 282: Speech Communication. Oldenburg (Deutschland), 2018.

[16] VAN DER OORD, A., DIELEMAN, S., ZEN, H., et al. WaveNet: A generative model for raw audio. ArXiv preprint, arXiv: $1609.03499 \mathrm{v} 2$ [cs.SD], 2016, p. 1-15.

[17] WANG, N., CHOI, J., BRAND, D., et al. Training deep neural networks with 8-bit floating point numbers. In Proceedings of the 32nd Conference on Neural Information Processing Systems (NeurIPS 2018). Montréal (Canada), 2018, p. 1-10.

[18] BANNER, R., HUBARA, I., HOFFER, E., et al. Scalable methods for 8-bit training of neural networks. In Proceedings of the 32nd Conference on Neural Information Processing Systems (NeurIPS 2018). Montréal (Canada), 2018, p. 1-9.

[19] SCHUMAKER, L. Spline Functions: Basic Theory. New York, USA: Cambridge University Press, 2007. ISBN: 978-0521705127

[20] GAROFOLO, S., et al. TIMIT Acoustic-phonetic Continuous Speech Corpus. Philadelphia, USA: Linguistic Data Consortium, 1993.

[21] ALEKSIC, D., PERIC, Z., NIKOLIC, J. Support region determination of the quasi-logarithmic quantizer for Laplacian source. Przeglad Elektrotechniczny, 2012, vol. 88, no. 7, p. 130-132. ISSN: 0033-2097

[22] VELIMIROVIC, L., MARIC, S. New adaptive compandor for LTE signal compression based on spline approximations. ETRI Journal, 2016, vol. 38, no. 3, p. 463-468. DOI: 10.4218/etrij.16.0115.0506

[23] NIKOLIC, J., PERIC, Z., VELIMIROVIC, L. Simple solution for designing the piecewise linear scalar companding quantizer for Gaussian source. Radioengineering, 2013, vol. 22, no. 1, p. 194-199. ISSN: 1210-2512

[24] HAMMAD, H., KHAN, M. N., GILANI, S. O., et al. H.264 encoder parameter optimization for encoded wireless multimedia transmissions. IEEE Access, 2018, vol. 6, p. 22046-22053. DOI: 10.1109/ACCESS.2018.2824835

[25] INTERNATIONAL TELECOMMUNICATION UNION. ITU-T G.712: Transmission Performance Characteristics of Pulse Code Modulation (PCM), 1992. 
[26] GAZOR, S., ZHANG, W. Speech probability distribution. IEEE Signal Processing Letters, 2003, vol. 10, no. 7, p. 204-207. DOI: 10.1109/LSP.2003.813679

\begin{abstract}
About the Authors ...
Zoran PERIC was born in Niš, Serbia, in 1964. He received the B.S., M.S. and Ph.D. degrees from the Faculty of Electronic Engineering, University of Niš, Serbia, in 1989, 1994 and 1999, respectively. He is a full professor at the Department of Telecommunications, Faculty of Electronic Engineering, University of Niš. His current research interests include: the digital communications, signal processing and information theory. $\mathrm{He}$ is an author and coauthor of over 260 papers. Dr. Perić has been a reviewer in a number of journals, including IEEE Transactions on Information Theory, IEEE Transactions on Signal Processing, IEEE Transactions on Communications, Digital Signal Processing, Compel, Informatica, Information Technology and Control, Expert Systems with Applications and IEEE Signal Processing Letters.
\end{abstract}

Jelena NIKOLIC was born in Prokuplje, Serbia, in 1978. She received the B.S., M.S. and Ph.D. degrees in Electrical Engineering from the Faculty of Electronic Engineering, University of Niš, Serbia in 2003, 2006 and 2011, respectively. She is currently an Assistant Professor at the De- partment of Telecommunications at the same faculty. Her expertise is in the field of digital communications and compression. Her current research interests include source coding, quantization, compressed sensing, artificial intelligence and machine learning. She has published over 100 papers, 44 of them have been published in journals from SCI list. She was a reviewer for numerous reputable journals. She is an Editor of the Journal of Advanced Computer Science \& Technology.

Bojan DENIC (corresponding author) was born in Strpce, Serbia, in 1986. He received B.S. and M.S. degrees in Electronics and Telecommunications from the Faculty of Technical Sciences, University of Priština, Serbia. He is currently a research assistant and Ph.D. student at the Faculty of Electronic Engineering, University of Niš, Serbia. His current research interests include scalar quantization and signal processing. He has published several papers in peer-reviewed international journals on the above subject.

Vladimir DESPOTOVIC received his Ph.D. degree in Electrical Engineering from the University of Niš, Serbia, in 2012. Currently he is working as an Associate Professor at the University of Belgrade, Serbia. Previously he was engaged as postdoctoral researcher at the University of Paderborn, Germany. His main research interests include statistical signal processing, natural language processing, speech coding, fractional calculus and machine learning. 